\title{
Molecular and cytogenetic characterization of highly repeated DNA sequences in the vole Microtus cabrerae
}

\author{
ROSA FERNÁNDEZ†, MARÍA JOSÉ L. BARRAGÁN‡, MÓNICA BULLEJOS $\ddagger$, JUAN \\ ALBERTO MARCHAL + SERGIO MARTÍNEZ $\$$, RAFAEL DÍAZ DE LA GUARDIA§ \\ \& ANTONIO SÁNCHEZ*\$ \\ $\uparrow$ Departamento de Psicobiología, Facultad Ciencias de la Educación, Universidad de A Coruña, E-15071 A Coruña, \\ Spain, $\$$ Departamento de Biología Experimental, Facultad de Ciencias Experimentales y de la Salud, Universidad de \\ Jaén, E-23071 Jaén, Spain and §Departamento de Genética, Facultad de Ciencias, Universidad de Granada, E-18071 \\ Granada, Spain
}

\begin{abstract}
The genus Microtus presents several species with extremely large sex chromosomes that contain large blocks of constitutive heterochromatin. Several cytogenetic and molecular studies of the repetitive sequences in species of the genus Microtus have demonstrated that the heterochromatin is highly heterogeneous. We have cloned and characterized a family of repetitive DNA sequences from M. cabrerae, a species with large heterochromatic blocks on the giant sex chromosomes. These repetitive sequences are $65.84 \% \mathrm{~A}-\mathrm{T}$ rich, organized in tandem, with a 161-bp unit and are located on the centromeric region of autosomes and the $\mathrm{X}$ chromosome. In addition, this repetitive DNA is located throughout the entire heterochromatic block of the $\mathrm{X}$ chromosome and on three interstitial bands in the heterochromatic block of the $\mathrm{Y}$ chromosome. Comparative analysis of this family of repetitive sequences from three Microtus species revealed that the development of these sequences has occurred by concerted evolution. Our results support the hypothesis that the heterochromatic blocks from the sex chromosomes of different species are evolving independently and they probably have the genetic capacity to amplify and retain different satellite DNAs. For a topic related to the location of these repetitive DNA sequences on the $\mathrm{Y}$ chromosome of $M$. cabrerae, we propose a model to explain the origin of a length polymorphism previously described for this chromosome.
\end{abstract}

Keywords: constitutive heterochromatin, Microtus cabrerae, repetitive DNA, satellite DNA, sex chromosomes.

\section{Introduction}

Mammalian genomes are characterized by a large number of repetitive sequences, which are highly variable from one species to another. Two main types of highly repetitive sequences are known in mammalian genomes: interspersed DNA, in which the repeated DNA sequences are dispersed throughout the genome; and satellite DNA, which is characterized by long tandem arrays and consistent association with constitutive heterochromatin (Singer, 1982). The localized repeats, or satellite DNAs, are typically found at C-band-positive regions of chromosomes, such as centromeres, telomeres and portions of the $\mathrm{Y}$ chromosome (Modi et al., 1988; Hamilton et al., 1990). The satellite DNAs are organized in tandem arrays of repeat units

\footnotetext{
*Correspondence. E-mail: abaca@ujaen.es
}

which are generally $\mathrm{A}-\mathrm{T}$ rich and show high variability affecting monomer size, nucleotide sequence, copy number, and genome and chromosome organization and localization (Charlesworth et al., 1994).

The biological function of these DNA sequences remains elusive, although it has been proposed that they are involved in centromeric condensation, sister chromatid pairing, chromosome association with the mitotic spindle, karyotypic evolution and chromosome arrangement (Miklos \& John, 1979; Singer, 1982; Vogt, 1992).

The genus Microtus presents several species with extremely large sex chromosomes which contain large blocks of constitutive heterochromatin (Sperling et al., 1987; Burgos et al., 1988; Modi, 1993a,b). Several cytogenetic and molecular studies of repetitive sequences in Microtus species have demonstrated that the heterochromatin is highly heterogeneous, as several unrelated 
repetitive sequences located in the heterochromatin of the sex chromosomes of Microtus chrotorrhinus and Microtus agrestis have been found (Modi, 1993a,b,c; Ivanov \& Modi, 1996; Kalscheuer et al., 1996; Neitzel et al., 1998). Furthermore, it has been shown that a satellite DNA from Microtus chrotorrhinus (MSAT160), shared with other Microtidae species, is evolving by concerted evolution (Modi, 1993b).

Hence, studies of repetitive sequences in other Microtus species are interesting, especially in species with large blocks of constitutive heterochromatin located on the giant sex chromosomes, where we can study the composition, location and evolution process in large amounts of heterogeneous heterochromatin.

Microtus cabrerae is a rodent species with a diploid number of $2 n=54$. The karyotype of this species is characterized by giant sex chromosomes (Diaz de la Guardia et al., 1979). In this species the constitutive heterochromatin is located on the sex chromosomes and on the centromeric regions of autosomes. Cytogenetic studies with C- and G-banding techniques, as well as the use of fluorochromes with different specificity, have demonstrated that the constitutive heterochromatin of M. cabrerae is highly heterogeneous (Burgos et al., 1988, 1990; Modi, 1993a). In fact, six classes of heterochromatin have been described in the sex chromosomes of this species according to its response to the above mentioned techniques (Burgos et al., 1988).

In this study we have cloned and characterized a family of repetitive DNA sequences from $M$. cabrerae. These repetitive sequences are $65.84 \%$ A-T rich, organized in tandem, with a 161-bp unit and located on the centromeric region of autosomes and the $\mathrm{X}$ chromosome. In addition, this repetitive DNA is located along the entire heterochromatic block of the X chromosome as well as on three interstitial bands in the heterochromatic block of the Y chromosome. Analysis of this family of repetitive sequences in three Microtus species revealed that the evolution of these sequences has occurred by concerted evolution. In relation to the location of these repetitive DNA sequences on the Y chromosome of $M$. cabrerae we propose a model to explain the possible involvement of these sequences in the origin of the length polymorphism previously described for this chromosome.

\section{Materials and methods}

\section{Animals, and DNA extraction}

Male and female individuals of four species from the genus Microtus were analysed. These species are Microtus cabrerae, M. arvalis, M. nivalis and M. agrestis. Genomic DNA was extracted from spleen cells according to standard phenol-chloroform procedures (Sambrook et al., 1989).

\section{Chromosomes, C-banding and Fluorescence in situ hybridization (FISH)}

M. cabrerae chromosomes were obtained following the method of Burgos et al. (1986), and C-band staining was performed by the technique of Sumner (1972) with slight modifications.

The FISH procedure followed was essentially that of Pinkel et al. (1986). Briefly, the slides were incubated in $100 \mathrm{~g} / \mathrm{mL}$ RNase $/ 2 \times \mathrm{SSC}$ solution at $37^{\circ} \mathrm{C}$ for $60 \mathrm{~min}$ and dehydrated in an increasing series of ethanol. The DNA of the cells was denatured in $70 \%$ formamide/ $2 \times \mathrm{SSC}$ at $70^{\circ} \mathrm{C}$ for 2 min and then dehydrated.

The probe (clone Mca-T67) was labelled with biotin16-dUTP (Roche) by PCR (Lo et al., 1990). Hybridization was carried out in a volume of $20 \mu \mathrm{L}$ of $50 \%$ formamide, $10 \%$ dextran sulphate, $5 \mathrm{mg}$ herring-sperm DNA, in $2 \times \mathrm{SSC}(\mathrm{pH}=7)$ for $16 \mathrm{~h}$ at $37^{\circ} \mathrm{C}$ in a moist chamber. Post-hybridization washes were at $40^{\circ} \mathrm{C}$ in $40 \%$ formamide $/ 2 \times \mathrm{SSC}$ and finally at $37^{\circ} \mathrm{C}$ in $2 \times \mathrm{SSC}$, three times for $5 \mathrm{~min}$ each. Slides were then blocked for $30 \mathrm{~min}$ at $37^{\circ} \mathrm{C}$ in $4 \times \mathrm{SSC} / 5 \%$ blocking reagent $(4 \mathrm{M})$ and were next incubated in the detection solution (FITC)-conjugated avidin (Oncor) at $37^{\circ} \mathrm{C} 30 \mathrm{~min}$ followed by three washes ( 5 min each) in $4 \times \mathrm{SSC} /$ $0.05 \%$ Tween $20(4 \mathrm{~T})$. For signal amplification slides were incubated in the antibody solution (biotinylated goat antiavidin, Oncor) and in a second round of avidin-FITC treatment. Slides were stained with 4',6-diamidino-2-phenylindole (DAPI).

Images were collected with a Nikon E-800 microscope equipped with a Hamamatsu CCD camera. Gray-scale images for either FITC or DAPI filter sets were pseudocoloured and further processed with AnalySIS 2.11.005 software.

\section{Cloning and sequencing}

Repetitive sequences of $M$. cabrerae present a typical ladder pattern when genomic DNA is digested with HinfI. The resulting fragments were separated on $2 \%$ agarose gel and stained with ethidium bromide. The monomeric unit of the ladder was $161 \mathrm{bp}$.

Bands corresponding to monomer, dimer and trimer were eluted from the gel and, after blunt end treatment with klenow enzyme, ligated into SmaI-digested pUC19 vector. In addition, monomer and dimer bands were also ligated with pGEM-T vector (Promega) as described by Sánchez et al. (1996). Briefly, the eluted DNA with $5^{\prime}$ overhang was treated with $1 \mathrm{U}$ of $T a q$ polymerase (Roche) using standard buffer and 2 mm of each dNTP, 
at $70^{\circ} \mathrm{C}$ for $2 \mathrm{~h}$ in a $20-\mu \mathrm{L}$ reaction volume to fill the gap and generate $3^{\prime}$ overhang ends with a single dATP. Seven microlitres of the resulting product were used in a normal ligation reaction with $50 \mathrm{ng}$ of pGEM-T vector, according to the supplier's instructions.

E. coli JM109 competent cells were transformed with the ligation reactions, and the recombinant bacteria containing the sequence of interest were selected after screening, using as probe the same dimer DNA band used for cloning, labelled with digoxigenin by random priming (Roche).

The positive clones were sequenced in both directions using the Thermosequenase fluorescent cycle sequencing kit from Amersham. The sequence reactions were analysed on a $6.5 \%$ polyacrylamide gel in a LICOR$400 \mathrm{~L}$ automated sequencer.

\section{Southern blot}

Genomic DNAs were digested with the appropriate restriction endonucleases. The fragments were separated in a 2\% agarose gel and blotted onto Nylon membranes (Amersham) according to Sambrook et al. (1989). Membranes were probed with eluted dimer band or Mca-T67 clone $(25 \mathrm{ng} / \mathrm{mL}$ of digoxigenin-labelled DNA) at $55^{\circ} \mathrm{C}$ overnight, and alkaline phosphatase detection was carried out according to the supplier's recommendation (Roche).

\section{Sequence analysis}

Pairwise sequence alignment and multiple alignment were carried out with the program CLUSTAL w 1.6 (Thompson et al., 1994). Genetic distances were calculated according to Kimura's (1980) two-parameter method, and the resulting distance matrices were subjected to neighbour-joining method analysis (Saitou \& Nei, 1987). A consensus tree was constructed, and the significance of the phylogenetic lineages was assessed by bootstrap analysis with 1000 replications. The phylogenetic analysis were carried out using the program MEGA version 1.02 (Kumar et al., 1993).

\section{Results}

Digestion of genomic DNA from $M$. cabrerae, $M$. agrestis, $M$. arvalis and $M$. nivalis with HinfI and electrophoresis results in a typical ladder pattern only in M. cabrerae (Fig. 1a). The size of the monomer unit is $161 \mathrm{bp}$ in length. When genomic DNA is digested with HinfI and probed with a digoxigenin-labelled dimer band, a regular ladder pattern can be seen in M. cabrerae (Fig. 1b). The size of each band corresponds to the size of multimers of the monomer unit. Although by

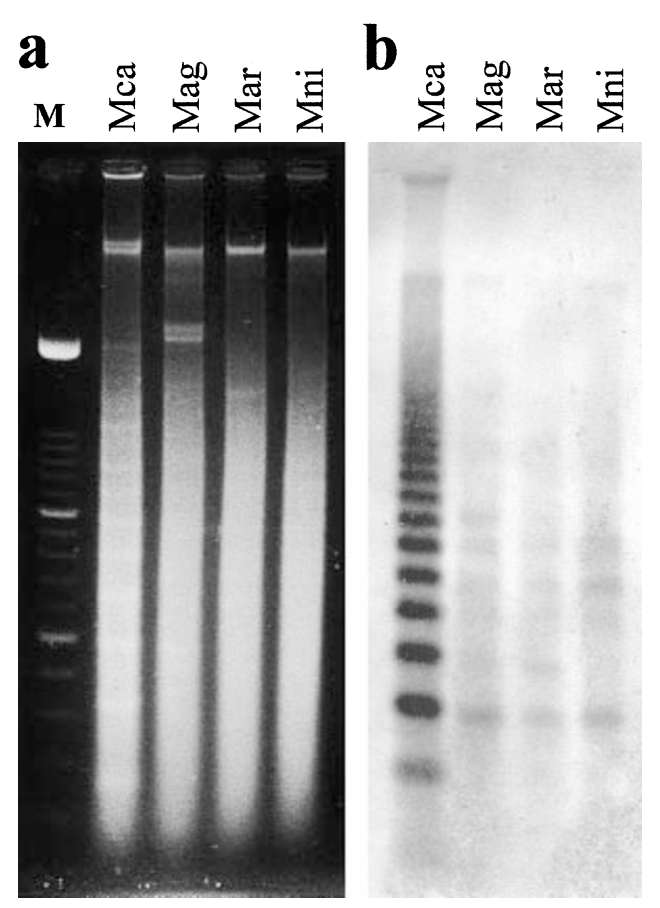

Fig. 1 (a) Gel electrophoresis of HinfI digested genomic DNA from M. cabrerae (Mca), M. agrestis (Mag), M. arvalis (Mar) and $M$. nivalis (Mni). M, 100-bp molecular weight marker (Roche). (b) Southern blot of the same gel probed with the random primer digoxigenin-labelled dimer band.

Southern blot the presence of a ladder pattern can be seen in the genome of the other Microtus species analysed, the hybridization signals are less intense when compared to the intensity of the band observed in M. cabrerae. This may be due to lower copy numbers of these sequences in the mentioned species. Furthermore, in all these species but M. cabrerae, the most represented unit is the dimer repeat, and the monomer is absent when genomic DNAs are digested with HinfI.

After ligation with SmaI digested pUC-19 vector, transformation and screening, two positive clones were obtained: (i) monomer 12 (Mca-M12), which although cloned from a monomer band, incorporated two monomer units (named Mca-M12a and b); and (ii) trimer 67 (Mca-T67), which incorporates a trimer repeat unit.

The remaining positive clones were monomers (McaM1, 4, 66, 67) and dimers (Mca-D8, 9, 10, 32, 45), obtained after a ligation reaction using the pGEM-T vector (Promega). The monomer units contained in each dimer or trimer have been named as units ' $a$ ', ' $b$ ' and 'c' (examples: Mca-D8a and 8b for dimer 8 and Mca-T67a, $67 \mathrm{~b}$ and $67 \mathrm{c}$ for trimer 67 ).

Sequences from different clones showed that the satellite DNA cloned in $M$. cabrerae belongs to the 
same repetitive family as that described in $M$. chrotorrhinus and M. agrestis (Modi, 1992; 1993b). Figure 2(a) shows the alignment of 19 different units cloned from $M$. cabrerae, 10 complete monomer units from M. chrotorrhinus (Modi, 1992) and the only monomer sequence described in M. agrestis (Modi, 1993b). All sequences cloned from $M$. cabrerae were $161 \mathrm{bp}$ in length, with the exception of Mca-D9b which was $160 \mathrm{bp}$ due to a deletion mutation and Mca-T67c which was $9 \mathrm{bp}$ shorter, possibly as a consequence of the blunt end treatment, as is demonstrated by the absence of a target sequence for HinfI in one end of that clone.

The consensus sequence for cloned repetitive DNA in $M$. cabrerae was $65.84 \% \mathrm{~A}-\mathrm{T}$ rich. The percentage of
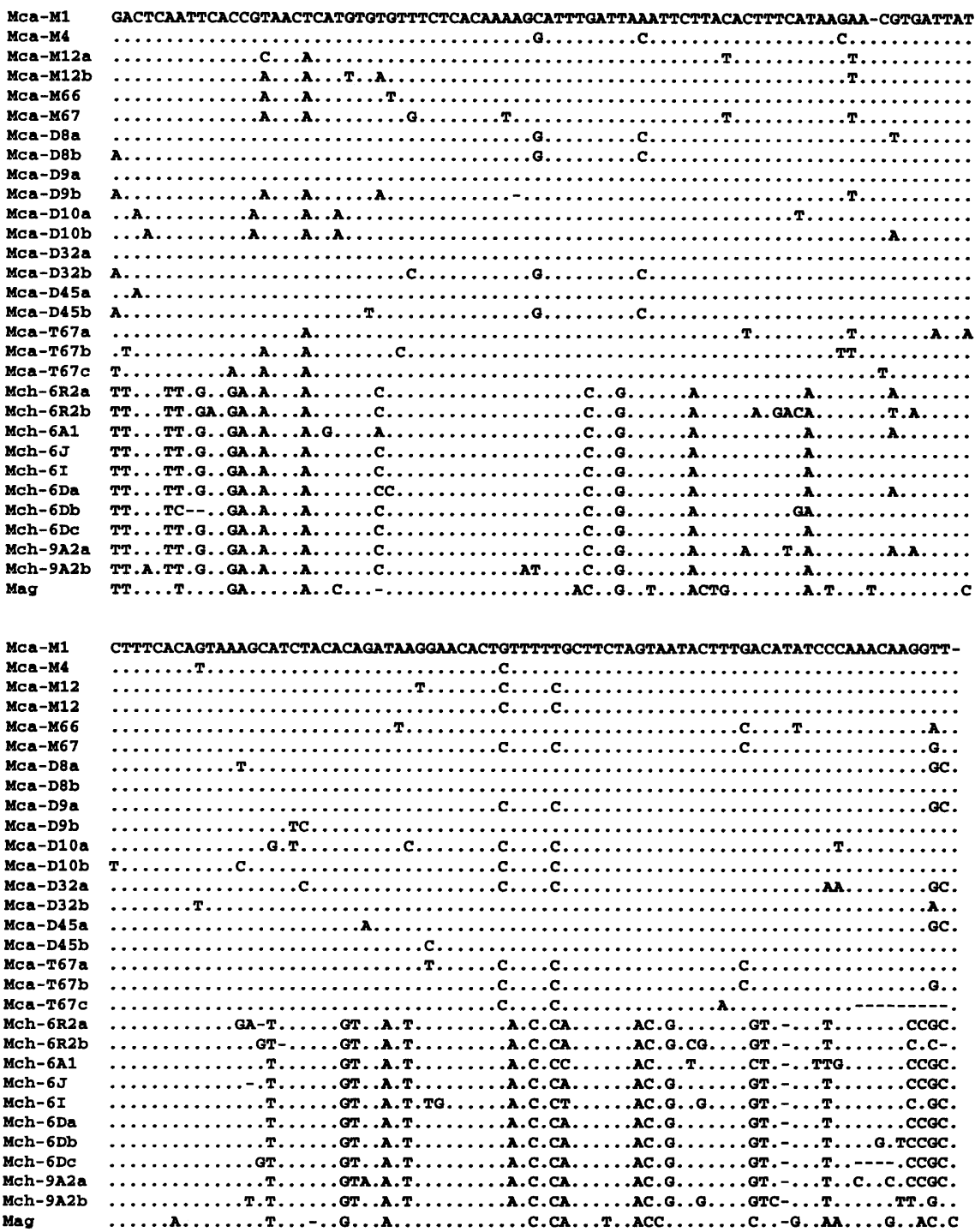

b

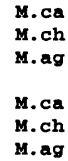

GACTCAATTCACCGTAACACATGTGTGTTTCTCACANAAGCATTTGATтANATTCTTACACTTTCATAAGAA-CGTGATTAT

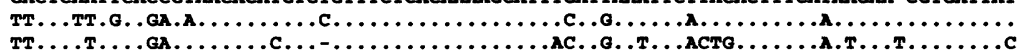

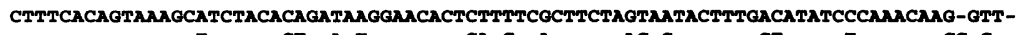

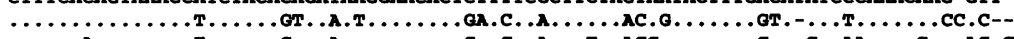

Fig. 2 (a) Sequence alignment of the 19-monomer unit cloned from $M$. cabrerae (Mca), the 10-monomer unit of MSAT-160 from M. chrotorrhinus (Mch) and the one-monomer unit of this satellite from $M$. agrestis (Mag). M. cabrerae sequences have been submitted to GenBank and have been assigned the accession numbers from AJ311756 to AJ311767. (b) Alignment of the consensus sequence of satellite DNA from $M$. cabrerae with the consensus sequence of MSAT-160 from $M$. chrotorrhinus and with the exclusive reported sequence of this satellite from $M$. agrestis. Data of satellite sequences of $M$. chrotorrhinus and $M$. agrestis are from Modi (1992, 1993b). 
identity of the different monomer units with the consensus sequence ranged between $94.4 \%$ and 98.13\%. Different G-A transitions (McaD8, 9, 32 and 45), a T-A transversion (Mca-D10) and G-T and A-T transversions (Mca-T67) were responsible for the dimer and trimer formation as a consequence of the loss of target sequences for HinfI. Although mutation events were homogeneously distributed, $71.7 \%$ of the changes occurring in monomers were transversions and $28.3 \%$ were transitions. This implies a transversion-to-transition ratio of 2.5 , slightly higher than the expected value of 2 for random base changes.

Figure 2(b) shows the alignment of the consensus sequence of the repetitive DNA from $M$. cabrerae with the consensus sequences of the repetitive DNA from M. chrotorrhinus (MSAT-160) (Modi, 1993b) and with the only monomer sequence reported for $M$. agrestis (Modi, 1993b). The consensus sequence from M. cabrerae shows percentages of identity of $79.87 \%$ and $75.60 \%$ with the sequences from $M$. chrotorrhinus and $M$. agrestis, respectively.

The methylation status of this repetitive DNA was analysed in the two species with giant sex chromosomes containing large blocks of heterochromatin. The genomic DNA, from both males and females, was digested independently with the methylation-insensitive enzyme $M s p I$ and with the methylation-sensitive enzyme HpaII. After Southern blot the membrane was probed with Mca-T67 labelled with digoxigenin. In $M$. agrestis the Southern analysis showed the same hybridization pattern in males and females. However, there were differences between both enzymes, as in the two sexes $M s p I$ digestion gave rise to bands that were lost with HpaII digestion. This result indicates that some cytosines in CCGG target sites are methylated in this species. On the other hand, in M. cabrerae there were differences between males and females when hybridization patterns were compared. Although no differences existed in relation to the enzyme used in female DNA, in males a clear band appeared in the Southern only when the enzyme used was $M s p I$, and this band was absent when HpaII was used for digestion. This result implies that there is some methylation in CCGG target sequences that occur exclusively on the male genome and hence these sequences are probably in the $\mathrm{Y}$ chromosome (Fig. 3).

Fluorescent in situ hybridization with the clone McaT67 localized this repetitive DNA in the centromeric regions of autosomes and the $\mathrm{X}$ chromosome, which are heterochromatic as demonstrated by C-banding (Fig. 4a,b). Furthermore, there was hybridization on the entire heterochromatic region of the $\mathrm{X}$ chromosome, although in this case the signal intensity was less than the centromeric signal. The Y chromosome showed no

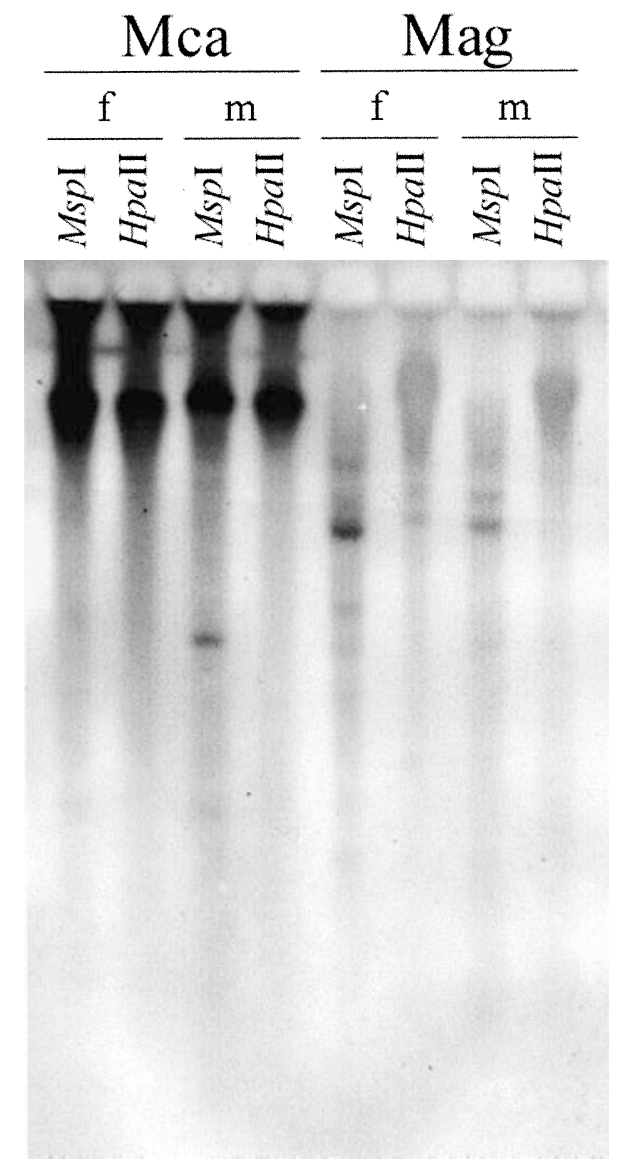

Fig. 3 Southern blot of M. cabrerae (Mca) and M. agrestis (Mag) female (f) and male (m) genomic DNA, digested with the methylation-insensitive enzyme $M s p I$ and with the methylation-sensitive enzyme HpaII, probed with the digoxigenin-labelled clone Mca-T67.

signal in the centromeric region but three faint interstitial bands in the heterochromatic region (Fig. 4c).

A neighbour-joining tree using the Kimura-2 parameter distance was constructed with 19 monomer units from $M$. cabrerae, 10 complete monomer units from $M$. chrotorrhinus (Modi, 1992) and the only described sequence from M. agrestis (Modi, 1993b) (Fig. 5). Although most of the internal branches were poorly supported, those that discriminate between species monomers were well supported. All monomer units from $M$. cabrerae are clearly located (100 bootstrap value) on a branch separated from those belonging to $M$. chrotorrhinus or $M$. agrestis. Furthermore, the sequences for these two later species are also split into two separate branches with a bootstrap value of 96 . In fact, the CLUSTAL alignment of the monomer units from these species showed the existence of multiple 

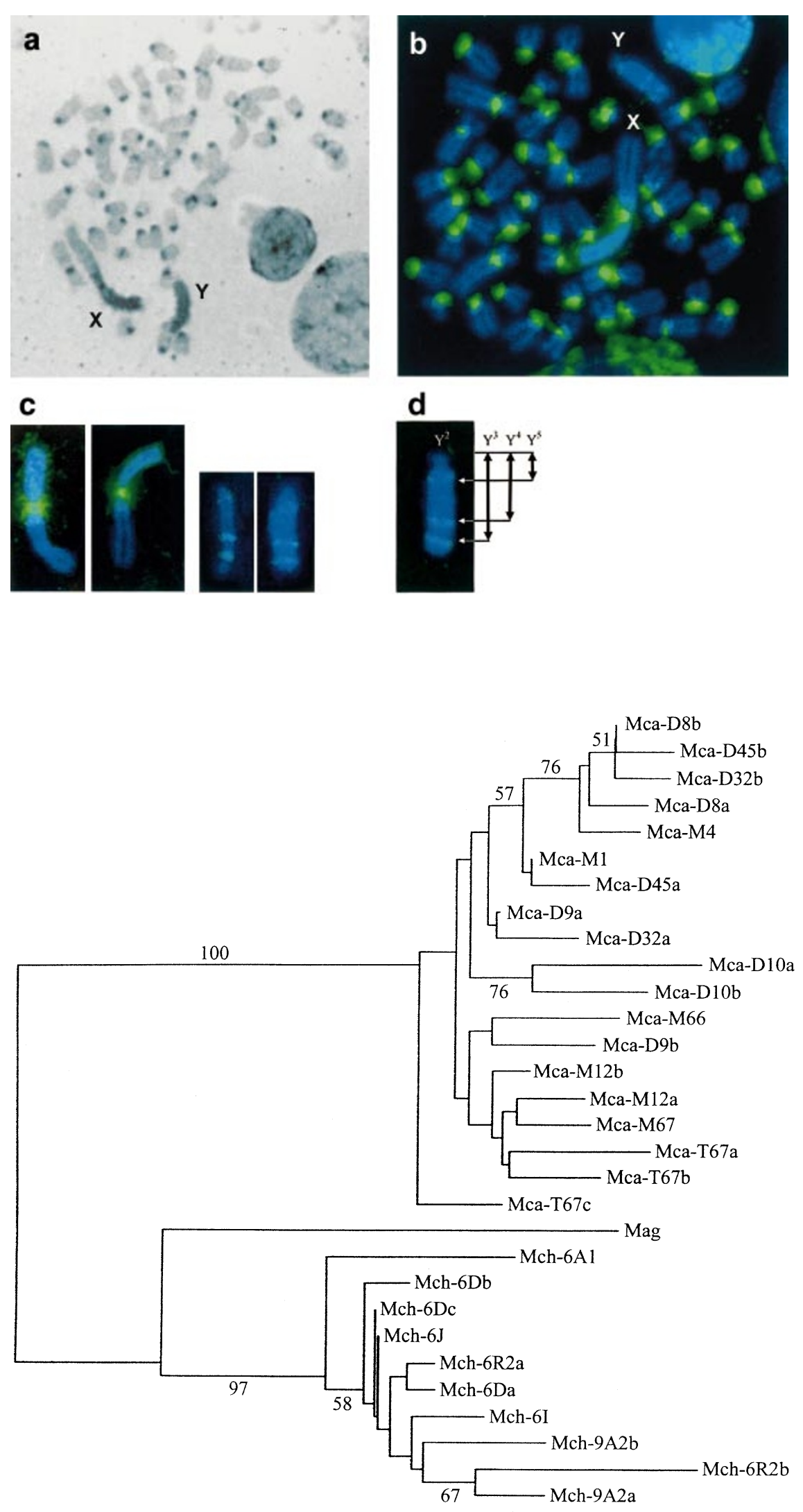

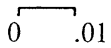

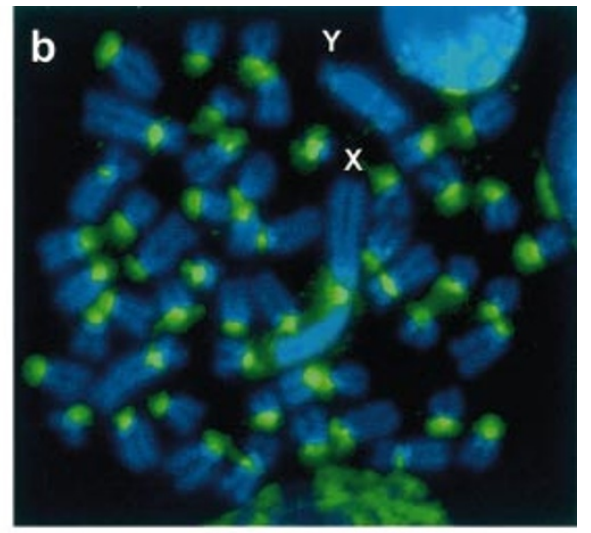

d

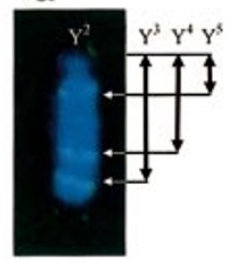

Fig. 4 (a) C-banded chromosomes from a $M$. cabrerae male individual. (b) Fluorescence in situ hybridization with cloned repeat DNA on male metaphase chromosomes from M. cabrerae. (c) Selected X and Y chromosomes hybridized as in $\mathrm{b}$. (d) Y chromosome with a schematic drawing indicating the possible breakage points in the hybridized repetitive band which could give rise to the $\mathrm{Y}^{3}, \mathrm{Y}^{4}$ and $\mathrm{Y}^{5}$ chromosomes.

Fig. 5 Neighbour-joining dendrogram based on genetic distances (using Kimura's two-parameter method) between the repetitive units of satellite DNA from M. cabrerae (Mca), M. chrotorrhinus (Mch) and $M$. agrestis (Mag). The values in the branches indicate the bootstrap probabilities based on 1000 replicates. Only the bootstrap values over 50 are shown. The branch length is drawn to the scale of the genetic distances (shown at the bottom). 
diagnostic positions, that is, species-specific changes (Fig. 2a,b).

\section{Discussion}

The genus Microtus is a rodent group from the Arvicolidae family which contains about 60 species. Some of the species from this genus present giant sex chromosomes. M. cabrerae, as other species in this group, in addition to general heterochromatic pericentromeric regions on the autosomes, presents large blocks of heterogeneous constitutive heterochromatin located on the giant sex chromosomes. This characteristic makes this species an interesting model in which to study repetitive DNA sequences.

Electrophoresis analysis carried out on genomic DNA from $M$. cabrerae digested with HinfI has shown ladder pattern bands, indicating the presence of repeated sequences in the genome of this species. In eukaryotic genomes, highly repeated sequences generally occur in two main patterns, repeated sequences dispersed throughout the genome or clustered in tandem arrays. The presence of a ladder pattern in gels stained with ethidium bromide and in Southern blot probed with the digoxigenin-labelled dimer band clearly indicated that the repeat sequences in $M$. cabrerae are arrayed in tandem. The bands containing several monomer units originated by point mutations that modify the target sequences for HinfI, as has been demonstrated after sequencing dimer and trimer units.

Sequence alignment allowed the determination of the consensus sequence for the HinfI repeats, with a length of $161 \mathrm{bp}$. The A-T content of $M$. cabrerae repetitive DNA was $65.84 \%$, slightly higher than MSAT-160 from M. chrotorrhinus (64\%) (Modi, 1992) or satellite DNA from other rodent species, which range from $60 \%$ to $63 \%$ in A-T content (reviewed in Singer, 1982). A high A-T content is considered to be a general feature of satellite DNA (Singer, 1982). Comparative analysis of 19 monomer units demonstrated a high sequence homology, with base substitution mutations randomly spread along the sequences. The same substitutions occurred in several clones of this satellite DNA at the same position in the sequences. Such a mutational pattern could be explained as the result of homogenization processes; by unequal crossing-over and amplification, some particular monomer variations can be spread throughout the whole satellite (Dover, 1986). The evolution of satellite DNA is mainly determined by processes of concerted evolution. The concept 'concerted evolution' describes processes by which a sequence can be amplified, homogenized throughout the genome and distributed among both homologous and nonhomologous chromosomes (Dover, 1982). Several mechanisms have been proposed to be active in certain genomes, such as unequal crossing-over, gene conversion, sequence transposition and rolling circle replication (Dover, 1982; Walsh, 1987), which can act together or individually during the process of concerted evolution.

The repetitive DNA sequences cloned in $M$. cabrerae belong to a family of satellite DNA present in other Microtus species, as demonstrated by Southern blot analysis. In fact, this family of repetitive sequences has been described previously at the molecular level in M. chrotorrhinus and M. agrestis (Modi, 1992, 1993a,b,c). By Southern blot and by using in situ hybridization Modi (1993a,b) demonstrated that these sequences are present in the genome of all Microtus species he analysed. For these species in situ hybridization showed that this repetitive family is located in almost all centromeric regions and in other C-band positive regions of constitutive heterochromatin. In species of the genus Microtus with giant sex chromosomes the hybridization pattern of the satellite DNA on the sex chromosomes is species-specific. In $M$. chrotorrhinus this family of repetitive DNA hybridizes on the centromere of the $\mathrm{X}$ chromosome and not on the $\mathrm{Y}$ centromere. Also, the entire heterochromatic regions of both sex chromosomes are positive (Modi, 1993a). In $M$. agrestis, with the exception of the $\mathrm{X}$ chromosome centromere, no other hybridization signal is observed on the eu- or heterochromatic regions of the sex chromosomes (Modi, 1993a). In M. cabrerae, the repetitive DNA hybridizes intensely with the $\mathrm{X}$ chromosome centromere, but no signal is detected on the Y centromere. Also, signal can be observed on the entire heterochromatic region of the $\mathrm{X}$ chromosome, whereas on the heterochromatic region of the $\mathrm{Y}$ chromosome only three faint interstitial bands are present.

By C- and G-banding and fluorochrome staining, the heterochromatin of sex chromosomes of $M$. cabrerae has been demonstrated to be very heterogeneous (Burgos et al., 1988, 1990). According to the above mentioned techniques, Burgos et al. (1988) described the existence of six different types of heterochromatin in the sex chromosomes of this species, four on the $\mathrm{X}$ chromosome and two on the $\mathrm{Y}$ chromosome. The hybridization pattern of the repetitive sequences described here on the sex chromosomes of $M$. cabrerae also suggests that the heterochromatin of sex chromosomes is heterogeneous. The composition of the $\mathrm{Y}$ heterochromatin, deprived of the repetitive sequence described here, with the exception of the three interstitial bands, is different from the $\mathrm{X}$ heterochromatin. This does not mean that the heterochromatic block of these sex chromosomes does not share other repetitive DNA sequences. The heterochromatin of the X chromosome, including the centromeric region, is not homogeneous, 
because the centromeric region is mainly constituted of the repetitive sequences described here, whereas the remaining heterochromatin, which fluoresces faintly with these sequences, is constituted of other DNA sequences also. The presence of satellite DNA in the centromeres of autosomes and $\mathrm{X}$ chromosomes and its absence from the $\mathrm{Y}$ chromosome centromere is a common feature of satellite DNAs from rodent species (Kunze et al., 1999).

In relation to the heterogeneity of the heterochromatin blocks in other Microtus species, in M. chrotorrhinus three unrelated repetitive sequences have been described (MSAT-21 bp, MSAT-160 bp and MSAT2570), all located on the heterochromatic region of the sex chromosomes (Modi, 1992; 1993a,b,c; Ivanov \& Modi, 1996). In M. agrestis a repeat unit of $3 \mathrm{~kb}$ as well as L1 and non-L1 retroposons have been described, which are accumulated on the heterochromatic region of the sex chromosomes (Kalscheuer et al., 1996; Neitzel et al., 1998).

The molecular results about the repetitive DNA of $M$. cabrerae are consistent with previous cytogenetic reports. It is located on C-band regions of constitutive heterochromatin on autosome centromeres, which have been demonstrated to be A-T rich using Hoechst 33258 (Modi, 1993a). However, Burgos et al. (1990) showed that these centromeric regions do not fluoresce intensely when stained with DAPI, and some of them are CMA positive, that is, $\mathrm{G}-\mathrm{C}$ rich. In relation to the sex chromosome heterochromatin, Burgos et al. (1988) showed that the heterochromatic blocks of both $\mathrm{X}$ and $\mathrm{Y}$ chromosomes are DAPI positive, which is consistent with the high A-T content of the cloned repetitive DNA. For Y heterochromatin, in which only three bands can be detected after hybridization with these repetitive sequences, we can suppose that other $\mathrm{A}-\mathrm{T}$ rich sequences form part of this heterochromatin.

Burgos et al. (1988) described in M. cabrerae a length polymorphism, caused by deletion mutations, affecting the heterochromatic block of both $\mathrm{X}$ and $\mathrm{Y}$ chromosomes. These authors described three different $\mathrm{X}$ chromosomes (the standard $\mathrm{X}: \mathrm{X}^{\mathrm{s}}$, and two deleted Xs: $\mathrm{X}^{\mathrm{d} 1}$ and $\left.\mathrm{X}^{\mathrm{d} 2}\right)$ and five different $\mathrm{Y}$ chromosomes $\left(\mathrm{Y}^{1}, \mathrm{Y}^{2}, \mathrm{Y}^{3}\right.$, $\mathrm{Y}^{4}, \mathrm{Y}^{5}$ ), with $\mathrm{Y}^{1}$ the biggest and $\mathrm{Y}^{5}$ the smallest. Determination of the relation between $\mathrm{Y}$ chromosome length and the length of the highest autosome $\left(\mathrm{A}_{1}\right)$ demonstrated that all the male individuals analysed in this study had $\mathrm{Y}^{2}$ chromosomes. The $\mathrm{X}$ chromosome was the standard for all males and females. In the analysis of the hybridization patterns on the $\mathrm{Y}^{2}$ chromosome with the $M$. cabrerae satellite DNA described here, where only three interstitial positive bands were detected, when we estimated the relation between the length of the $\mathrm{Y}$ chromosome from the end of short arm to each one of the interstitial bands of repetitive DNA and the highest autosome length, the resulting relations were those described by Burgos et al. (1988) for $\mathrm{Y}^{3}, \mathrm{Y}^{4}$ and $\mathrm{Y}^{5}$ chromosomes (Fig. 2c). Hence we propose that the breakage points that gave rise to the length polymorphism on $\mathrm{Y}$ chromosomes could be the positions of this repetitive DNA (Fig. 4d).

Because repetitive DNA sequences are known to be subject to hypermethylation, we investigated the methylation status at CCGG sequences. In M. cabrerae female no differences were found after digesting the genomic DNA with MspI and HpaII, indicating that CCGG sequences are probably not methylated. By restriction enzyme banding and in situ nick-translation Burgos et al. (1992) demonstrated that constitutive heterochromatin in the $\mathrm{X}$ chromosome of $M$. cabrerae is methylated at specific sequences. These contradictory results could be explained if there are other repeated sequences located on the $\mathrm{X}$ heterochromatin, as is probably the case. No conclusive explanation can be reached, as these enzymes digest the whole genome poorly in this species and hence, a small number of CCGG target sequences are available to discern the methylation status of $\mathrm{X}$ chromosome heterochromatin. When male genomic DNA was digested with the same isoschizomers we found that some regions of the male genome were differentially methylated. In conjunction with the results in females, we conclude that some CCGG sites related to this repetitive sequences are methylated in the male genome and are probably on the Y chromosome.

The analysis of the methylation status of CCGG sequences in $M$. agrestis showed no differences between males and females. However, in this species we can conclude that some CCGG sequences associated with this repetitive DNA are methylated in both sexes, as clear differences were found in the Southern pattern when genomic DNA was digested with enzymes with different sensitivity to DNA methylation.

Modi (1992) demonstrated that MSAT-160, belonging to the same repetitive family studied here, is not methylated either in vivo or in vitro in the male genome of $M$. chrotorrhinus. However, other repetitive DNA (MSAT-2570) from the heterochromatic region of the sex chromosomes of the same species showed tissuespecific methylation. Furthermore, the $3-\mathrm{kb}$ repetitive sequences of $M$. agrestis, which are located in the heterochromatic region of the sex chromosomes, and are also present in the $M$. cabrerae genome and other Microtus species, are hypermethylated (Kalscheuer et al., 1996). In conclusion, the methylation status of the heterochromatic region of sex chromosomes in Microtus species depends on the species, sex and type of repeat sequence. 
Phylogenetic inference revealed that genetic distances between repeat units of the same species were smaller than genetic distances between repeat units from different species. As a consequence, neighbour-joining methods grouped the repeat by affinity and clearly separated the monomer unit of the three species under phylogenetic study into separate branches, with a bootstrap value of $100 \%$ for the branch that separated monomers of $M$. cabrerae and a $96 \%$ bootstrap value for the separation between $M$. chrotorrhinus and $M$. agrestis monomer units. These data suggest a concerted mode of evolution for this satellite DNA family. Hence, the repetitive DNA sequences described in $M$. agrestis, $M$. chrotorrhinus and M. cabrerae can be considered as paralogous sequences. Those sequences could have arisen by duplication or amplification in the ancestral species and have become slightly differentiated in each genome species. Concerted evolution has been demonstrated for other repetitive DNA from rodents, as in the case of the harvest mouse Reithrodontomys (Hamilton et al., 1990).

Microtus species with giant sex chromosomes do not possess the same repetitive sequences, and the heterochromatic blocks are evolving independently, hence it is possible that they have the genetic capacity to amplify and retain different DNA repeats in the sex chromosomes. This can be related to the fact that in M. cabrerae and $M$. agrestis, meiotic asynapsis of the sex chromosomes has been reported (Wolf et al., 1988; Jiménez et al., 1991) and accumulation of very highly repeated sequences is expected to occur in regions with very low recombination rates and weak selective constraints on array length (Charlesworth et al., 1994).

The genus Microtus is thought to have radiated about 1.6 million years ago (Chaline \& Graf, 1988). Different species contain different repetitive sequences and when the same repetitive sequence is in several species, the monomer units are more related to others belonging to the same species than to monomers from other species. These results imply that Microtus genomes are susceptible to rapid amplification of different repetitive sequences and independent concerted evolution.

The percentages of divergence between the consensus sequences of Fig. 2(b) are 19-25\%. This is a very large divergence for satellite DNA for a species radiation that occurred 1.6 million years ago, even with the high rodent evolutionary rates and the lack of evolutionary constraint on these sequences. Thus two possibilities exist, that the rate of evolution of this sequences is unexpectedly very high or that the ancestor of the three species presented a diverse collection of already different sequences, and after species separation different subsets of this collection became amplified in each species.
In conclusion, the constitutive heterochromatin of the sex chromosomes of Microtus species with large sex chromosomes is highly complex in structure and composition. In fact, it comprises several unrelated and related satellite DNA families that show species-specific methylation patterns, distribution and karyotypic localization, as demonstrated by both cytogenetic and molecular techniques.

\section{Acknowledgements}

We would like to thank the Junta de Castilla y León and the Junta de Castilla-La Mancha for capture permits for Microtus specimens. This work was supported by the Spanish DGESIC through project numbers: PB98-1378C02-01 and PB98-1378-C02-02, and by the Junta de Andalucía through the programme 'Ayudas a grupos de investigación’, group numbers: CVI 0109 and CVI 220.

\section{References}

BURGOS, M., JIMÉNEZ, R. AND DIAZ DE LA GUARDIA, R. 1986. A rapid, simple and reliable combined method for Gbanding mammalian and human chromosomes. Stain Technol., 61, 257-260.

BURGOS, M., JIMÉNEZ, R., OLMOS, D. M. AND DIAZ DE LA GUARDIA, R. 1988. Heterogeneous heterochromatin and size variation in the sex chromosomes of Microtus cabrerae. Cytogenet. Cell Genet., 47, 75-79.

BURGOS, M., JIMÉNEZ, R., SÁNCHEZ, A. AND DIAZ DE LA GUARDIA, R. 1992. Restriction enzyme banding and in situ nick-translation on different types of hetero- and euchromatin. Exp. Cell Res., 202, 545-548.

BURGOS, M., OLMOS, D. M., JIMÉNEZ, R., SÁNCHEZ, A. AND DIAZ DE LA GUARDIA, R. 1990. Fluorescence banding in four species of Microtidae: an analysis of the evolutive changes of the constitutive heterochromatin. Genetica, 81, 11-16.

CHALINE, J. AND GRAF, J. D. 1988. Phylogeny of the Arvicolidae (Rodentia): Biochemical and paleontological evidence. J. Mammal, 69, 22-33.

CHARLESWORTH, B., SNIEGOWSKI, P. AND STEPHAN, w. 1994. The evolutionary dynamics of repetitive DNA in eukaryotes. Nature, 371, 215-220.

DiAZ DE LA GUARDiA, R., PASCUAL, L. AND OROZCO, J. C. 1979. The karyotype of Microtus cabrerae Thomas, another species with giant sex chromosomes. Experientia, 35, 741.

DOVER, G. A. 1982. Molecular drive: a cohesive mode of species evolution. Nature, 199, 111-117.

DOVER, G. A. 1986. Molecular drive in multigene families: how biological novelties arise, spread and are assimilated. Trends Genet., 168, 159-165.

HAMILTON, M. J., HONEYCUTT. R. L. AND BAKER, R. J. 1990. Intragenomic movement, sequence amplification and concerted evolution in satellite DNA in harvest mice (Reithrodontomys): evidence from in situ hybridization. Chromosoma, 99, 321-329. 
IVANOV, S. V. AND MODI, W. S. 1996. Molecular characterization of the complex sex-chromosome heterochromatin in the rodent Microtus chrotorrhinus. Cytogenet. Cell Genet., 75, 49-56.

JIMÉNEZ, R., CARNERO, M., BURGOS, M., SÁNCHEZ, A. AND DIAZ DE LA GUARDIA, R. 1991. Achiasmatic giant sex chromosomes in the vole Microtus cabrerae (Rodentia, Microtidae). Cytogenet. Cell Genet., 57, 56-58.

KALSCHEUER, V., SINGH, A. P., NANDA, I., SPERLING, K. AND NEITZEL, H. 1996. Evolution of the gonosomal heterochromatin of Microtus agrestis: rapid amplification of a large, multimeric, repeat unit containing a $3.0-\mathrm{kb}(\mathrm{GATA})_{11}$-positive, middle repetitive element. Cytogenet. Cell Genet., 73, 171178.

KIMURA, M. 1980. A simple method for estimating evolutionary rates of base substitution through comparative studies of nucleotide sequences. J. Mol. Evol., 16, 111-120.

KUMAR, S., TAMURA, K. AND NEI, M. 1993. MEGA: Molecular evolutionary genetics analysis. Version 1.01. The Pennsylvania State University, University Park, PA.

KUNZE, B., TRAUT, W., GARAGNA, S., WEICHENHAN, D., REDI, C. A. AND WINKING, H. 1999. Pericentric satellite DNA and molecular phylogeny in Acomys (Rodentia). Chromosome Res., 7, 131-141.

LO, Y.-M. D., MEHAL, W. Z. AND FLEMING, K. A. 1990. Incorporation of biotinylated dUTP. In: Innis, M. A., Gelfand, D. H., Sninsky, J. J. and White, T. J. (eds) PCR Protocols: A Guide to Methods and Applications, pp. 113-118. Academic Press, San Diego, CA.

MIKLOS, G. L. AND JOHN, B. 1979. Heterochromatin and satellite DNA in man: properties and prospects. Am. J. Hum. Genet., 31, 264-280.

MODI, w. S. 1992. Nucleotide sequence and genomic organization of tandem satellite array from the rock vole Microtus chrotorrhinus (Rodentia). Mammalian Genome, 3, 226-232.

MODI, w. S. 1993a. Comparative analysis of heterochromatin in Microtus: sequence heterogeneity and localized expansion and contraction of satellite DNA arrays. Cytogenet. Cell Genet., 62, 142-148.

MODI, w. S. 1993b. Heterogeneity in the concerted evolution process of tandem satellite array in Meadow Mice (Microtus). J. Mol. Evol., 37, 48-56.

MODI, W. S. 1993c. Rapid, localized amplification of a unique satellite DNA family in the rodent Microtus chrotorrhinus. Chromosoma, 102, 484-490.
MODI, W. S., FANNING, T. G., WAYNE, R. K. AND O'BRIEN, S. J. 1988. Chromosomal localization of satellite DNA sequences among 22 species of felids and canids (Carnivora). Cytogenet. Cell Genet., 48, 208-213.

NEITZEL, H., KALSCHEUER, V., HENSCHEL, S., DIGWEED, M. AND SPERLING, K. 1998. Beta-heterochromatin in mammals: evidence from studies in Microtus agrestis based on the extensive accumulation of L1 and non-L1 retroposons in the heterochromatin. Cytogenet. Cell Genet., 80, 165-172.

Pinkel, D., Straume, T. AND GRAY, J. W. 1986. Cytogenetic analysis using quantitative, high-sensitivity, fluorescence hybridization. Proc. Nat. Acad. Sci. U.S.A., 83, 2934-2938.

SAITOU, N. AND NEI, M. 1987. The neighbor-joining method: a new method for reconstructing phylogenetic trees. Mol. Biol. Evol., 4, 406-425.

SAMBROOK, J., FRITSCH, E. F. AND MANIATIS, T. 1989. Molecular Cloning. A Laboratory Manual, 2nd edn. Cold Spring Harbor Laboratory, Cold Spring Harbor, NY.

SÁNCHEZ, A., BULLEJOS, M., BURGOS, M., JIMÉNEZ, R. AND DÍAZ DE LA GUARDIA, R. 1996. An alternative to blunt-end ligation for cloning DNA fragments with incompatible ends. Trends Genet., 12, 44.

SINGER, M. L. 1982. Highly repeated sequences in mammalian genomes. Int. Rev. Cytol., 76, 67-112.

SPERLING, K., KALSCHEUER, V. AND NEITZEL, H. 1987. Transcriptional activity of constitutive heterochromatin in the mammal Microtus agrestis (Rodentia, Cricetidae). Exp. Cell Res., 173, 463-472.

SUMNER, A. T. 1972. A simple technique for demonstrating centromeric heterochromatin. Exp. Cell Res., 75, 304-306.

THOMPSON, J. D., HIGGINS, D. G. AND GIBSON, T. J. 1994. CLUSTAL w: improving the sensitivity of progressive multiple sequence alignment through sequence weighting, positionsspecific gap penalties and weight matrix choice. Nucl. Acids Res., 22, 4673-4680.

voGT, P. 1992. Code domains in tandem repetitive DNA sequence structures. Chromosoma, 101, 585-589.

WALSH, J. B. 1987. Persistence of tandem arrays: Implication for satellite and simple-sequence DNAs. Genetics, 115, 553-567.

WOLF, K. W., BAUMGART, K. AND WINKING, H. 1988. Meiotic association and segregation of the achiasmatic giant sex chromosomes in the male field vole (Microtus agrestis). Chromosoma, 97, 124-133. 